\title{
ALTERAZIONI DEL CAVO ORALE E QUALITÀ DELLA VITA NEL PAZIENTE SCLERODERMICO. ASPETTI CLINICI. CAPILLAROSCOPICI E CONDIZIONAMENTI PSICOLOGICI
}

\author{
Dott. Antonacci Anna, Dott. Abbinante Antonia, Dott. De Vito Danila, Dott. Praino Emanuela,
} Dott. Iannone Florenzo

KEYWORDS: sclerodermia, cavo orale, qualità della vita

\section{ABSTRACT}

La Sclerosi Sistemica è una malattia infiammatoria del tessuto connettivo caratterizzata da una risposta autoimmune che causa vasculopatia e accumulo di collagene nella pelle e negli organi interni.

Obiettivo: verificare gli effetti della malattia sul cavo orale del paziente sclerodermico e la sua influenza sulla qualità della vita, analizzando aspetti clinici, capillaroscopici e psicofisici.

A tal fine è stato condotto uno studio osservazionale su 25 pazienti affetti. Sono stati rilevati indici specifici per la valutazione dello stato di salute dento-parodontale, eseguita la capillaroscopia orale e somministrati test per verificare l>impatto della patologia sulla qualità della vita. E' stato impostato un protocollo preventivo-terapeutico per alleviare la sintomatologia presente e ritardare la progressione delle patologie orali.

La deformità delle mani, l'ipomobilità di lingua e labbra, la microstomi e la xerostomia, compromettono le condizioni di igiene orale dei pazienti sclerodermici . La presenza di ectasie, microemorragie e megacapillari alterano gravemente il microcircolo. La progressiva disabilità, i continui cambiamenti del loro aspetto e le sofferenze fisiche favoriscono il peggioramento della qualità della vita

Sulla base dei dati emersi, risulta fondamentale la collaborazione dell'igienista dentale con il reumatologo per l'attuazione di un piano personalizzato che consideri in modo prioritario il contesto bio-psico-sociale del paziente.

\section{INTRODUZIONE}

La sclerosi sistemica $(\mathrm{SSc})$, è una malattia infiammatoria del tessuto connettivo, caratterizzata da alterazioni della risposta immunitaria cellulare e umorale e da una caratteristica vasculopatia degli elementi del microcircolo e delle arterie muscolari di piccolo calibro. L'accumulo di collagene e altri componenti della matrice connettivale (fibrosi), si verifica, a carico della cute e dei vari organi interni: cuore, polmone, rene, articolazioni, tendini, muscoli e apparato gastroenterico dall'oro-faringe al retto $(1,2)$.

\section{Manifestazioni orali nel paziente sclerodermico Le alterazioni del cavo orale nel paziente scleroder- mico rappresentano una conseguenza diretta sia della sostituzione da parte del collagene dei normali tessuti, sia del deposito del collagene stesso intorno a struttu- re nervose e vascolari. \\ Le manifestazioni più frequenti, presenti in circa il $40 \%$ dei pazienti con Sclerosi Sistemica, che coin- volgono il cavo orale sono: \\ - xerostomia \\ - microstomia \\ - carie \\ - aumento del rischio di malattia parodontale \\ - erosione dello smalto \\ - riassorbimento mandibolare \\ - neuropatia del trigemino \\ - telangectasie del viso e delle mucose (14)}

La capillaroscopia orale nella Sclerosi Sistemica.

La capillaroscopia orale è un'indagine strumentale non invasiva fondamentale per la valutazione del microcircolo. Suscita grande interesse, per la possibilità di osservare in vivo vasi di piccole dimensioni grazie all'ausilio di un video-microscopio che, offre la possibilità di fotografare immagini (35).

La capillaroscopia rientra tra gli esami indispensabili per la diagnosi di vasculopatie e microangiopatie. Le lesioni anatomo-patologiche hanno un ruolo fondamentale nell'aggravamento di tali patologie. Le indagini strumentali, indispensabili per completare l'esame clinico, permettono la valutazione morfologico-funzionale delle condizioni del microcircolo distrettuali.

La capillaroscopia è un esame non invasivo, innocuo, ripetibile, dai costi relativamente contenuti con cui è possibile controllare in vivo ed in tempo reale lesioni vascolari, monitorando nel tempo l'andamento delle vasculopatie periferiche (36).

Le condizioni patologiche differiscono dalla normalità per la presenza di variazioni che interessano: il diametro, la lunghezza dei vasi, l'organizzazione micro circolatoria, la morfologia. Le cellule adibite alla difesa svolgono la loro funzione (apoptosi), grazie all'espressione di recettori della parete endoteliale. Alterazioni della apoptosi, comportano una maggiore vulnerabilità del soggetto. Il contatto di linfociti e plasmacellule con la parete vascolare conferma il coinvolgimento del parodonto (apparato di sostegno 


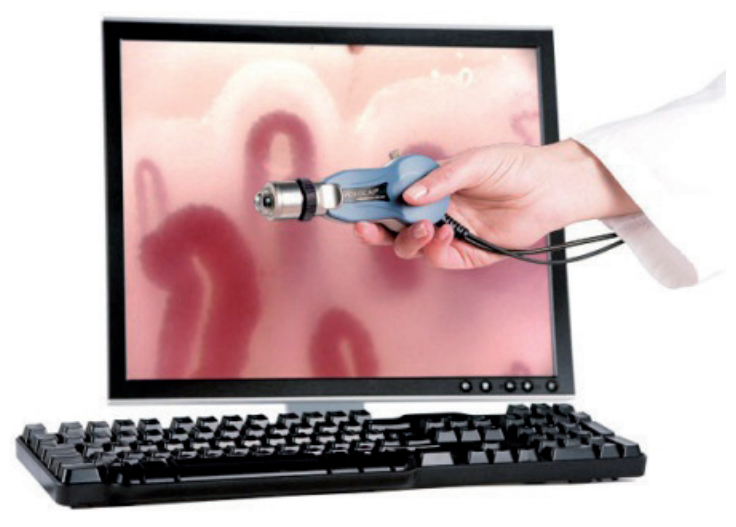

Figura 1. Videocapillaroscopio a sonda ottica e luce polarizzata.

del dente). Questi dati morfologici appaiono rilevanti in particolari patologie, come la Sclerosi Sistemica, dove le alterazioni capillari coinvolgono oltre al letto ungueale anche il microcircolo della mucosa parodontale. Queste variazioni sono fondamentali nella patogenesi e nel trattamento delle alterazioni parodontali nei pazienti con SSc $(37,47)$.

La classica unità capillare, meglio nota come unità microvascolare è costituita da:

- Una componente arteriosa che corrisponde alla porzione terminale del ramo arterioso periferico di piccolo calibro, con una parete estremamente sottile;

- Una componente capillare maggiormente coinvolta negli scambi trofico-metabolici tra sangue e tessuti. La parete dei capillari modula la propria permeabilità passiva in risposta alle sollecitazioni e ciascun capillare modifica il proprio calibro in seguito al passaggio di stato da sol a gel e viceversa, dei mucopolisaccaridi endocapillari.

- Una componente venosa, che può essere postcapillare e rappresenta una riserva della funzione capillare e collettrice, con un diametro crescente ed una parete ricca di cellule muscolari lisce.

- Incostante componente anatomica arteriovenulare che può essere di Tipo I costituito da connessioni dirette (shunts preterminali) ed un Tipo II più complicato per la presenza di un ulteriore segmento interposto fra quello arterioso e quello venoso,

- Componente perivascolare è infine costituita dai mastociti e prende legami con il sistema peri-linfatico dai capillari linfatici; anastomosi fondamentale poiché ha la funzione di regolare gli scambi fra sangue e tessuti e di garantire il drenaggio.

Il microcircolo presenta delle differenze di posizione, forma, calibro e microangiotettonica, con una variabilità interindividuale ampliamente dimostrata con la capillaroscopia.

\section{ANALISI DEL MICROCIRCOLO NELLA MUCOSA ORALE}

La capillaroscopia orale trova indicazione in tutte le patologie che comportano anomalie anatomiche e funzionali del microcircolo. Molti studi dimostrano come tale tecnica rappresenti un'interessante indagine complementare o alternativa alla capillaroscopia periungueale.
I vantaggi dell'indagine strumentale comprendono facilità e rapidità di accesso ed esecuzione, ridotta influenza di stimoli meccanici e chimici presenti a livello orale, minore sensibilità della mucosa orale nel confronti del freddo, assenza di invasività, basso costo e possibile valore predittivo di una patologia. I limiti possono riguardare difficoltà: di posizionamento della sonda, di immobilizzare la testa del paziente, movimenti dovuti alla respirazione, insufficiente visibilità dei capillari e variabilità del pattern cosiddetto "normale".

Con l'introduzione della tecnica della Videocapillaroscopia a Sonda ottica a Luce polarizzata sono state superate tutte le problematiche legate alle metodiche della capillaroscopia classica. Un esempio può essere la difficoltà di analizzare in maniera precisa alcune sedi anatomiche poiché l'obiettivo del capillaroscopio tradizionale rimane fisso e le variazioni spaziali del campo ottico richiedono il movimento della sede anatomica stessa, che ovviamente, risulta ampio e non controllabile. Tale difficoltà invece, non sussiste con la videocapillaroscopia in cui è l'obiettivo che si sposta, a piacimento dell'osservatore.

L'impiego di questa nuova tecnica ha permesso di esplorare distretti poco conosciuti, documentando come i capillari delle diverse regioni cutanee e/o mucose differiscano per posizione, calibro, forma e microangiotettonica. La digitalizzazione ha inoltre garantito la standardizzazione dei parametri quali diametro, lunghezza, morfologia dei vasi, la densità capillare ed il flusso ematico, consentendo di confrontarli nel tempo. Si deduce bene come questo esame può essere eseguito anche in campo odontoiatrico a livello delle mucose del cavo orale.

Da un'indagine bibliografica risulta che è necessario valutare le condizioni del microcircolo attraverso dati parametrici e dati non parametrici.

\section{DATI NON PARAMETRICI:}

- Visibilità delle anse: difficoltà di messa a fuoco (punteggio da 0 a 4);

- Orientamento rispetto alla superficie: (punteggio A, B, o AB). (A) andamento parallelo rispetto alla superficie, (B) andamento perpendicolare, $(\mathrm{AB})$ andamento sia parallelo che perpendicolare;

- Presenza di microemorragie: (Punteggio 0 o 1). (0) assenza, (1) presenza.

- Caratteristiche delle anse capillari: (punteggio 0 o 1). (0) assenza, (1) presenza.

\section{DATI PARAMETRICI:}

- Densità capillare: numero di anse presenti per unità di superficie;

- Calibro delle anse, (in genere è compreso tra i 4 ed i $14 \mu$ ed il valore è ottenuto dalla media di due osservazioni per ogni area controllata).

Entrambi i valori sono ottenuti dalla media di due osservazioni per ogni area (38)

\section{ASPETTI PSICOLOGICI DEL PAZIENTE SCLERODERMICO}

L' interessamento cutaneo e dei vari organi ed apparati, nei pazienti affetti da $\mathrm{SSc}$, è responsabile della minore aspettativa di vita e della sua alterata qualità. $\mathrm{Ai}$ comuni sintomi come: dolore, problematiche gastrointestinali, deformità articolare, limitazione nei 


\begin{tabular}{|c|c|c|c|}
\hline Non-parametric datn & Mark & Feature & Image \\
\hline \multirow{4}{*}{ Cupillary kop visisibility } & 1 & Simple fosusing & \\
\hline & 2 & Average focusing & \\
\hline & 3 & Diffícult Gressing & \\
\hline & 4 & Improssible ficusing & \\
\hline \multirow{3}{*}{$\begin{array}{l}\text { Orientation to the } \\
\text { surface }\end{array}$} & $\mathrm{A}$ & $\begin{array}{l}\text { Capillary kop course } \\
\text { paralkel to the surface }\end{array}$ & \\
\hline & B & $\begin{array}{l}\text { Capillary kop course } \\
\text { perpendiculur to the sur face }\end{array}$ & \\
\hline & AB & $\begin{array}{l}\text { Borth parallel and } \\
\text { perpendidicular }\end{array}$ & \\
\hline \multirow{2}{*}{ Miscrohemorthages } & a & Absence & \\
\hline & 1 & Preacnec & \\
\hline \multirow{2}{*}{$\begin{array}{l}\text { Characteristics of the } \\
\text { capillary loops }\end{array}$} & 0 & Absence & \\
\hline & 1 & Presence & \\
\hline
\end{tabular}

Figura 2.

movimenti, dispnea, disturbi del sonno; si associano disturbi psichiatrici, quali ansia e depressione, come conseguenza diretta della cronicità che caratterizza la patologia stessa. $(39 ; 40)$. Alti livelli di dolore cronico, stanchezza, insoddisfazione per il loro aspetto e disabilità generale, rendono i pazienti con SSc parti- colarmente a rischio per gli stati depressivi. La patologia spesso induce modifiche estetico-funzionali alle parti visibili del corpo (mani, bocca, viso), con insoddisfazione per la propria immagine così ampia da essere paragonata a quella dei pazienti con gravi ustioni. I pazienti affetti da SSc, comunemente segnalano difficoltà nel prendersi cura del loro aspetto, ad eseguire lavori domestici, portare avanti impegni lavorativi o partecipare ad attività per il tempo libero. La bocca ed il volto sono spesso coinvolti nello sviluppo della patologia e i pazienti spesso lamentano preoccupazioni estetiche, microstomia, riduzione ed alterazione della dentizione, ipomobilità della lingua e xerostomia, che possono ridurre 1' idoneità al lavoro (42).

Anche in Italia un'alta percentuale di pazienti sclerodermici $(33,4 \%)$ presenta sintomi depressivi classificabili da lievi a severi. Le manifestazioni depressive sono correlate alla percezione individuale della gravità di malattia. E' emerso come i soggetti che giudicano severo un qualsiasi aspetto della propria malattia, ad eccezione di quello gastroenterico, siano più a rischio di una deflessione dell'umore. I pazienti sclerodermici modificano frequentemente i propri rapporti sociali in ragione delle alterazioni fisiche conseguenti alla malattia, atteggiamento più evidente e marcato nelle persone giovani e in quelle depresse. Tale riscontro suggerisce la necessità di sviluppare indici e scale di valutazione oggettiva, sia delle alterazioni estetiche legate alla malattia che delle conseguenze cognitive e affettive, al fine di individuare soggetti a rischio con condotte di isolamento sociale e fornire loro un adeguato ascolto da parte del curante o di un opportuno supporto psicologico (43).

In questi pazienti spesso si riscontra sofferenza emo-
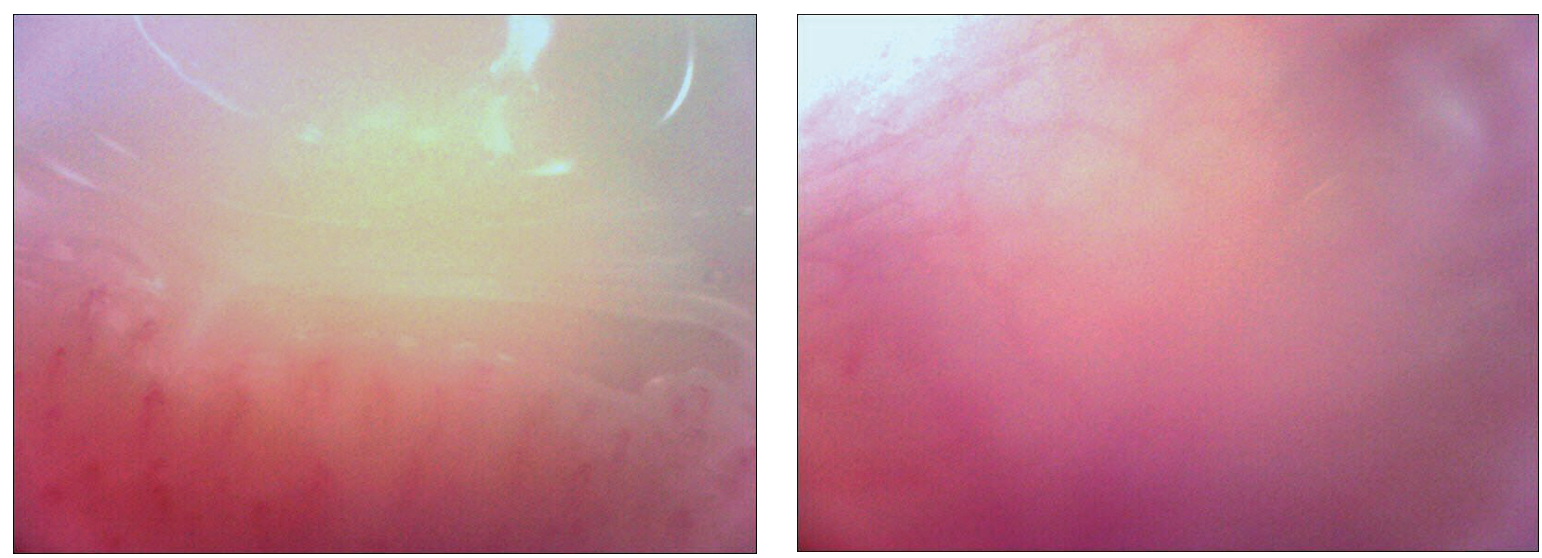

Figura 3. Capillaroscopia gengivale. Paziente sano (sinistra) e malato (destra) a confronto.
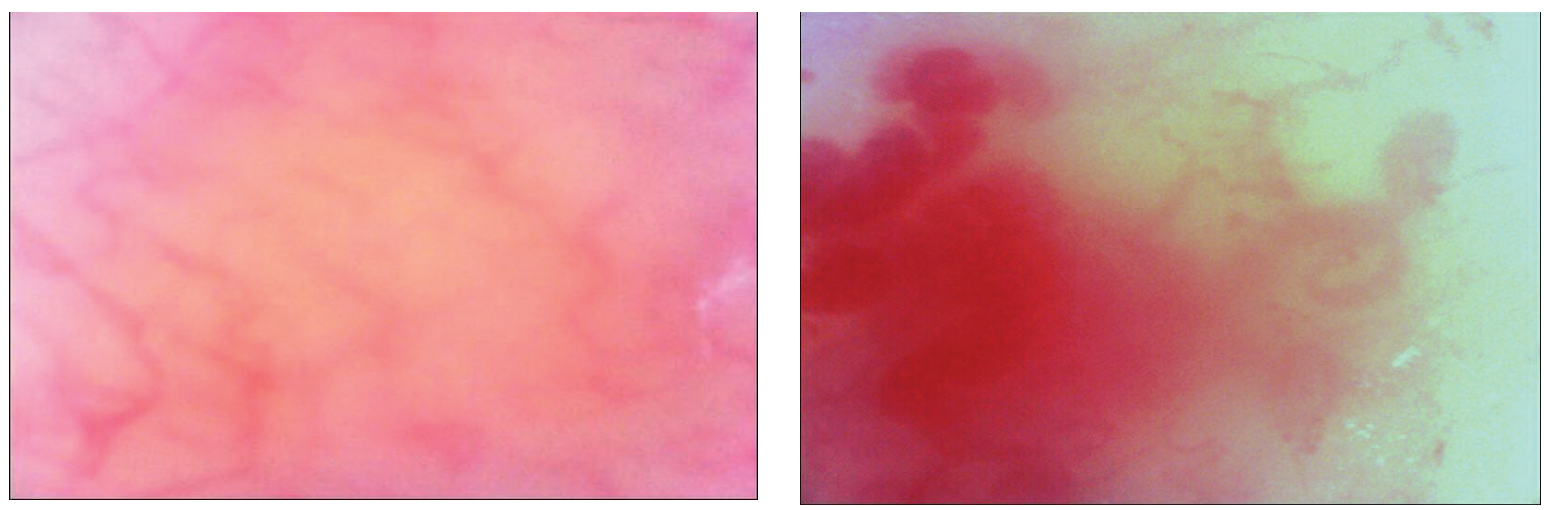

Figura 4. Capillaroscopia labiale. Paziente sano (sinistra) e malato (destra) a confronto. 
tiva, insoddisfazione di vita, maggiore utilizzo di assistenza sanitaria, comorbidità e mortalità, non solo come semplice conseguenza della malattia medica. I disturbi dell' umore hanno, infatti, il loro impatto sulla salute fisica, sia attraverso percorsi fisiologici che attraverso processi cognitivi, comportamentali e sociali. Sintomi di depressione sono associati ad una scarsa compliance ai regimi di trattamento medico, con probabilità ridotta di modificare comportamenti dannosi, come il fumo, l'isolamento sociale con minore probabilità di aumentare il sostegno sociale (41). Una corretta informazione multisciplinare fornita da reumatologo, dermatologo, medico generico psicologo o psichiatra, può mitigare l'ansia per il decorso della malattia, e migliorare il coping dei pazienti, ovvero la capacità di "cercare alleanza", "acquisire auto-responsabilità" e "pensare positivo". Queste abilità possono essere potenziate da gruppi di auto-aiuto, e nelle malattie rare e invalidanti come la Sclerosi Sistemica, i pazienti e le associazioni familiari possono promuovere il passaggio da una "malattia equilibrata" ad un "paziente equilibrato". L'intervento dello psichiatra, indispensabile in caso di gravi danni alla salute mentale, può essere rinviato o sostituito da auto-aiuto o da psicoterapia di sostegno, attraverso meccanismi che riducono la risposta allo stress.

L'eterogeneità della SSc, la sua gravità, e la varietà delle sue conseguenze su organi ed apparati, richiede un approccio multidisciplinare e un'alleanza tra pazienti ed operatori sanitari $(45 ; 46)$

\section{STUDIO OSSERVAZIONALE SULLE MANIFESTAZIONI ORALI NEL PAZIENTE SCLERODERMICO}

\section{SCOPO DEL LAVORO}

Scopo dello studio, verificare la presenza di alterazioni nel cavo orale del paziente sclerodermico e l'influenza della malattia sulla qualità della vita, analizzando aspetti clinici, capillaroscopici e psicofisici.

\section{OBIETTIVI}

Obiettivo primario: analizzare i dati clinici, le manifestazioni del cavo orale, le alterazioni vascolari del microcircolo; valutare il livello delle abilità funzionali manuali; verificare i livelli di ansia e depressione, al fine di mostrare l'influenza della Sclerodermia sul cavo orale e sulla qualità della vita.

Obiettivo secondario: impostare un protocollo preventivo/terapeutico personalizzato per alleviare la sintomatologia e ritardare la progressione delle patologie orali.

\section{MATERIALI E METODI}

A 25 pazienti con diagnosi di Sclerosi Sistemica afferenti al reparto di Reumatologia del Policlinico di Bari, di età compresa tra i 28 e 75 anni (età media 54 anni), è stata eseguita una completa anamnesi generale. E' stata indagata l'eventuale familiarità con patologie autoimmuni, l'epoca di insorgenza dei sintomi e l'anno di diagnosi. Sono stati raccolti i dati relativi alla terapia farmacologica attuale e pregressa (scheda di valutazione del paziente), i risultati della capillaroscopia periungueale e del Rodnan Skin thickness, atto a valutare il grado di ispessimento cutaneo del volto e delle mani.
Ai pazienti afferenti all'ambulatorio di Reumatologia per visita di controllo è stato somministrato un questionario, per raccogliere informazioni circa le abitudini relative all'igiene orale, agli stili di vita e alla presenza di manifestazioni orali, quali:

- sanguinamento gengivale,

- gengivite,

- malattia parodontale,

- mobilità dentale,

- ridotta secrezione salivare,

- alitosi,

- apertura limitata della bocca,

- retrazione delle labbra,

- respirazione orale,

- lesioni della mucosa orale,

- alterazioni della lingua,

- problemi articolari.

Sono stati quindi rilevati specifici indici per la valutazione dello stato di salute dento-parodontale:

- PCR indice di placca.

- PSR per la valutazione dello status parodontale.

- DMFT per verificare l'incidenza dei processi cariosi.

- BEWE per la valutazione del rischio di erosione dentale.

\section{TEST SALIVARE}

È stato eseguito un prelievo di saliva per la quantificazione del flusso e la determinazione del potere tampone attraverso strisce reattive a viraggio colorimetrico.

\section{MICROSTOMIA}

Il grado di microstomia è stato rilevato misurando la distanza intercommesurale, interincisale e di massima apertura orale.

\section{TEST DELLA FUNZIONALITÀ DEGLI ARTI SUPERIORI ED INFERIORI S-HAQ}

Il livello delle abilità funzionali (arti superiori e inferiori) è stato rilevato somministrando lo s-HAQ test, composto da 20 domande suddivise nelle diverse categorie di funzionamento (vestirsi, mangiare, camminare, curare l' igiene, ecc.) misurate su una scala da zero (nessuna difficoltà) a tre (no).

\section{CAPILLAROSCOPIA ORALE}

L'indagine capillaroscopica è stata condotta presso la sala visita del reparto di Reumatologia al mattino, sempre con la stessa fonte di luce, con pazienti in posizione seduta. Le aree indagate per ciascun paziente sono state gengiva marginale e mucosa labiale in corrispondenza degli incisivi inferiori. Sono stati analizzati:

1. Dati non parametrici: visibilità delle anse, orientamento rispetto alla superficie, presenza di microemorragie, caratteristiche delle anse capillari, densità capillare;

2. Dati parametrici: calibro delle anse e tortuosità.

à delle anse,

- 7 una media visibilità,

- 12 una densità capillare ridotta,

- 5 presentano microemorragie,

14 tortuosità delle anse,

- 11 ispessimento del tessuto gengivale. 


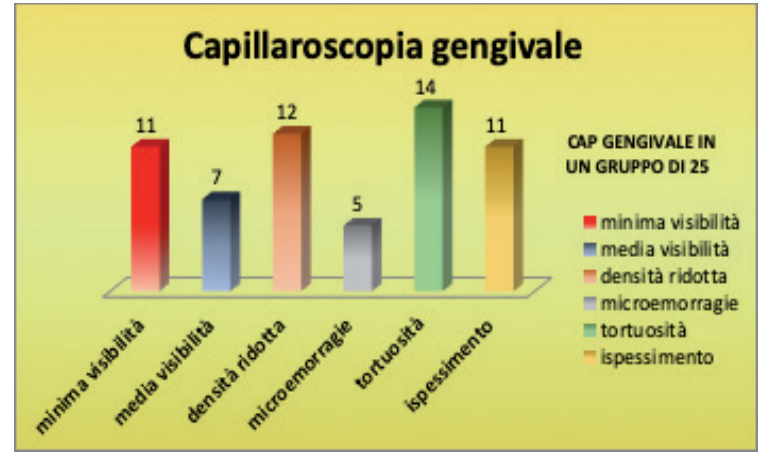

Figura 5.

- Capillaroscopia labiale:

- 9 presentano minima visibilità delle anse, 9 media visibilità,

4 presentano microemorragie,

16 capillari con un decorso tortuoso,

6 ispessimento tissutale per fibrosi,

5 pazienti presentano ectasie,

4 megacapillari,

5 rarefazione del reticolo.

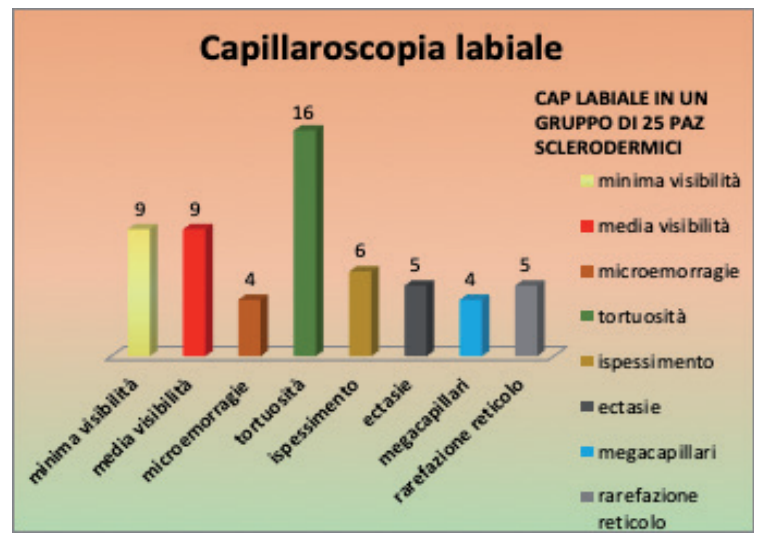

Figura 6.

- $\quad$ s-HAQ test, grado di disabilità:

- il $56 \%$ dei pazienti presenta un grado lieve,

- il $22 \%$ moderato,

- il $22 \%$ grave.

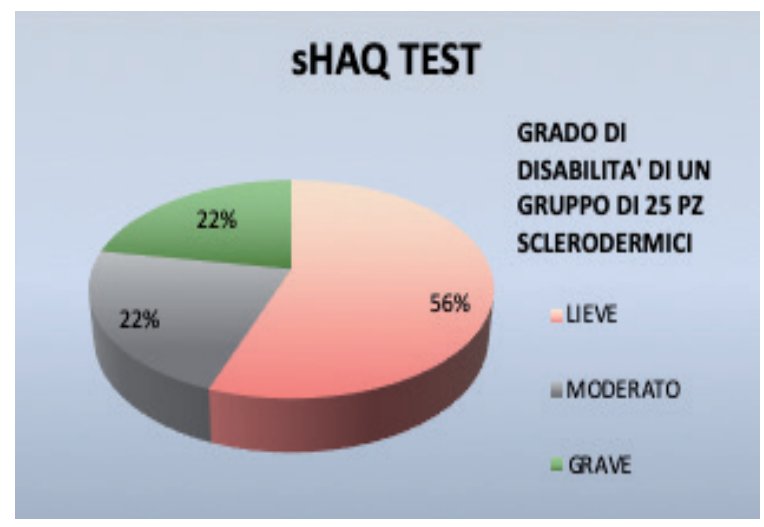

Figura 7.

- Test ansia (SAS):

- il 39\% dei pazienti presenta un grado di ansia basso,

○ il $44 \%$ un grado medio,

○ il $17 \%$ un alto grado

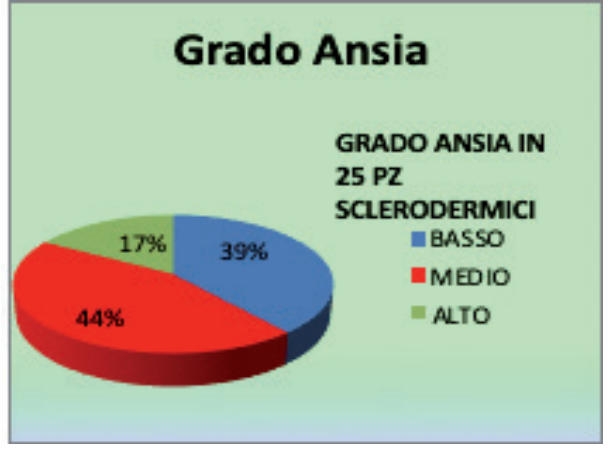

Figura 8.

○ Test depressione (SDS):

- il $28 \%$ dei pazienti mostra un grado di depressione basso,

○ il 55\% un grado medio,

○ il $17 \%$ un grado alto.

○

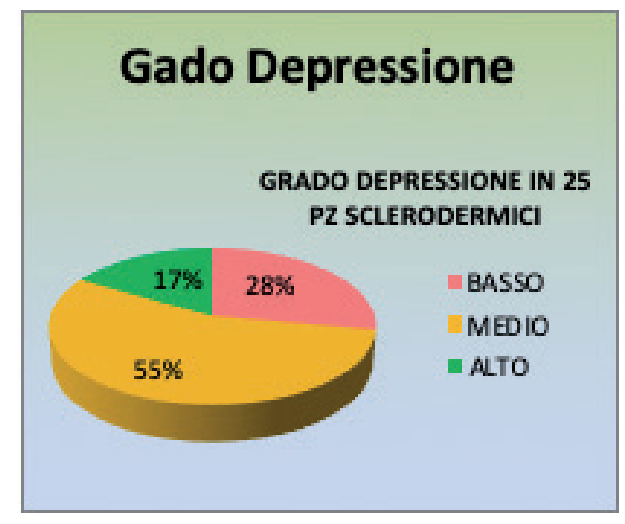

Figura 9.

- Test sulla qualità della vita WHOQOL-BREF Mediamente i 25 pazienti esaminati mostrano sulla scala di valutazione da 0 (bassa qualità della vita) a 100 (alta qualità della vita):

○ salute fisica: 32.3 ,

- stato psicologico 33.7,

- relazioni sociali 42.1

○ condizioni ambientali 32.3

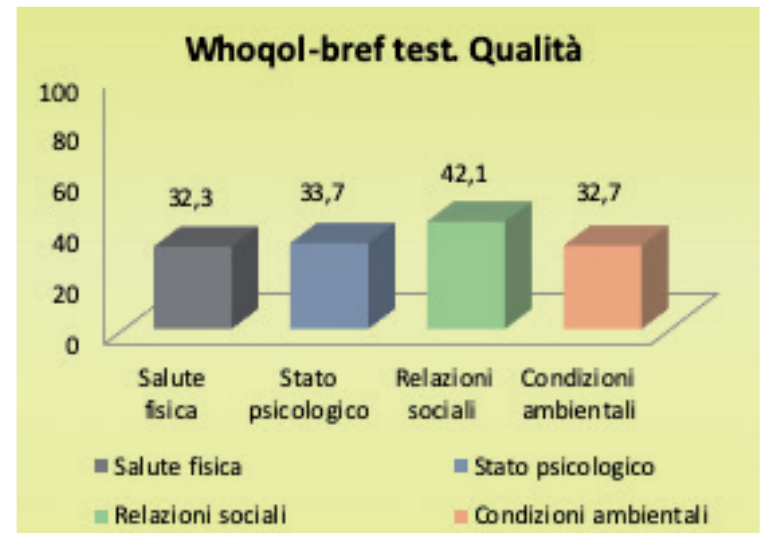

Figura 10. Bassa qualità della vita, 100: alta qualità della vita 
Si analizzano singolarmente le diverse aree.

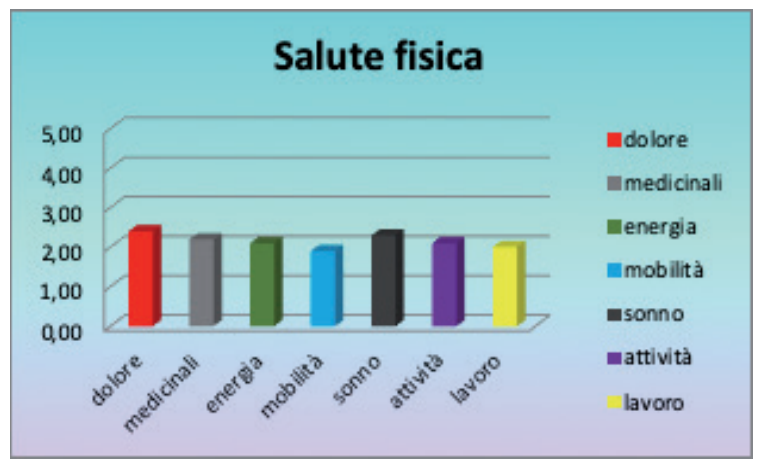

Figura 11. 0: bassa qualità della vita, 5: alta qualità della vita

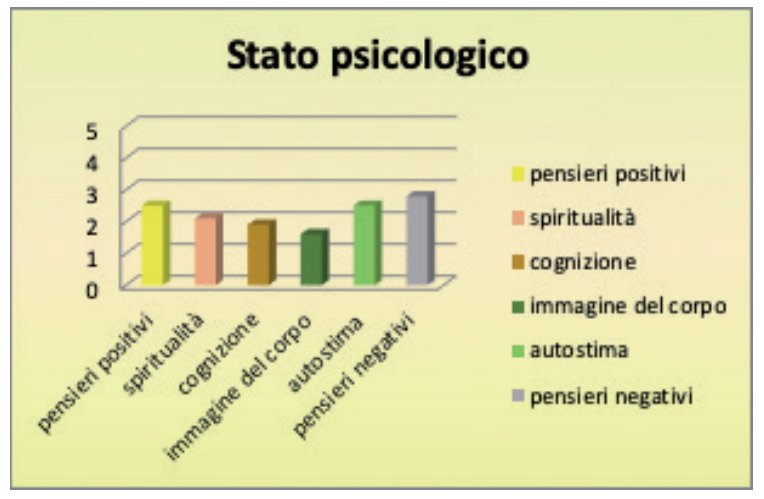

Figura 12. 0: bassa qualità della vita, 5: alta qualità della vita

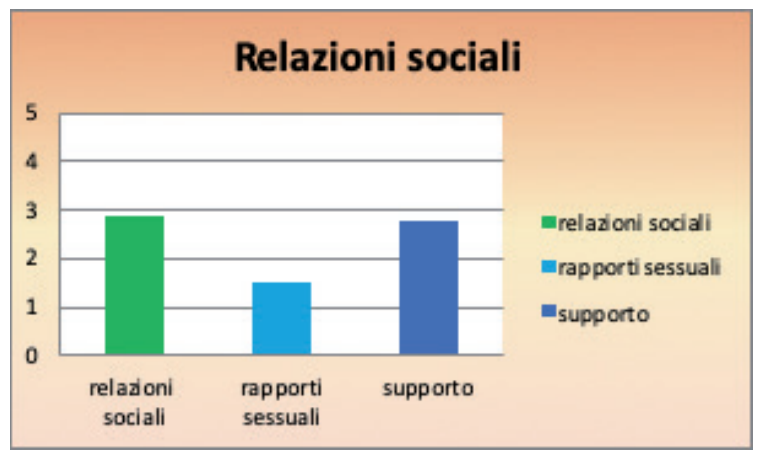

Figura 13. 0: bassa qualità della vita, 5: alta qualità della vita

\section{Confronto tra qualità della vita e stato di salute}

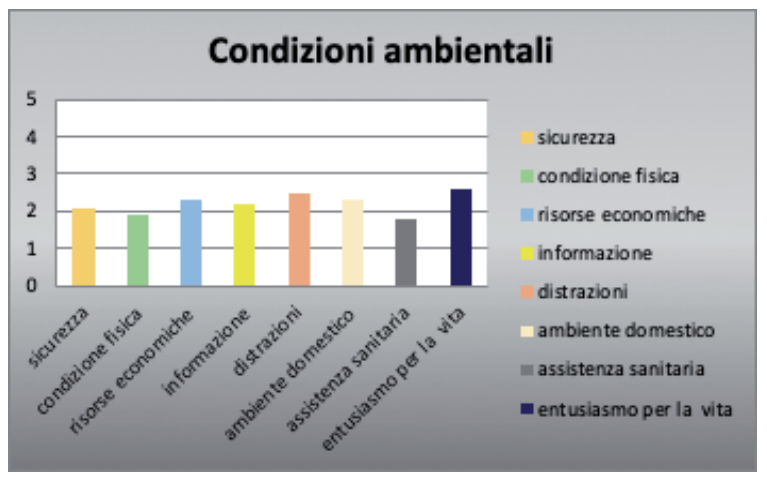

Figura 14. 0: bassa qualità della vita, 5: alta qualità della vita

\section{PROTOCOLLO DOMICILIARE}

Sulla base dei dati raccolti sono state impostate e somministrate in un successivo momento lezioni di igiene orale, istruzione e motivazione personalizzata. Somministrazione protocollo domiciliare :

- Prevenzione delle lesioni cariose, abrasioni:

- controlli periodici, sedute di igiene orale professionale,

- riduzione della frequenza di assunzione alimenti cariogeni/acidi, meticolosa igiene orale

- spazzolino manuale con filamenti medio/ morbidi (impugnatura ergonomica o con pallina), spazzolino elettrico.

- Trattamento della xerostomia/iposalivazione:

- sostituti/stimolanti salivari topici e sistemici, uso di collutori a base di clorexidina $\%$ 0.12 privi di alcool, sistemi polienzimatici in forma di gel, collutori e dentifrici

- assunzione sovente di acqua, uso di chewingum senza zuccheri aggiunti con enzimi, xilitolo, utilizzo di vaselina o burro cacao per idratare le labbra.

- Microstomia: stretching orale.

\section{DISCUSSIONI}

Lo studio osservazionale ha permesso di verificare che le condizioni di igiene orale nei pazienti sclerodermici, a supporto dello studio effettuato da Tolle S.L. nella School of Dental Hygiene USA 2008 (14), risultano compromesse. La maggior parte dei pazienti presenta un indice placca superiore al 50\%. spesso associato a gengivite, parodontite, sanguinamento e mobilità dentale.

Alla luce dei dati rilevati, queste condizioni sono dipese dalla ridotta autodetersione fisiologica, conseguenza della xerostomia, della ipomobilità della lingua, delle guance e delle labbra e da una ridotta igiene personale dovuta alle sclerodattilia ed alla microstomia.

A sostegno dello studio effettuato da Scardina e co. nel 2004, nel Dip. di Odontostomatologia dell'Università di Palermo (35), sono state riscontrate correlazioni tra alterazioni del cavo orale e compromissione vascolare parodontale. Il microcircolo si rivela compromesso per la presenza di ectasie, rarefazione del reticolo, tortuosità, microemorragie e megacapillari. Attraverso la sonda capillaroscopica è stato individuato in alcuni pazienti, anche uno stato di ispessi-

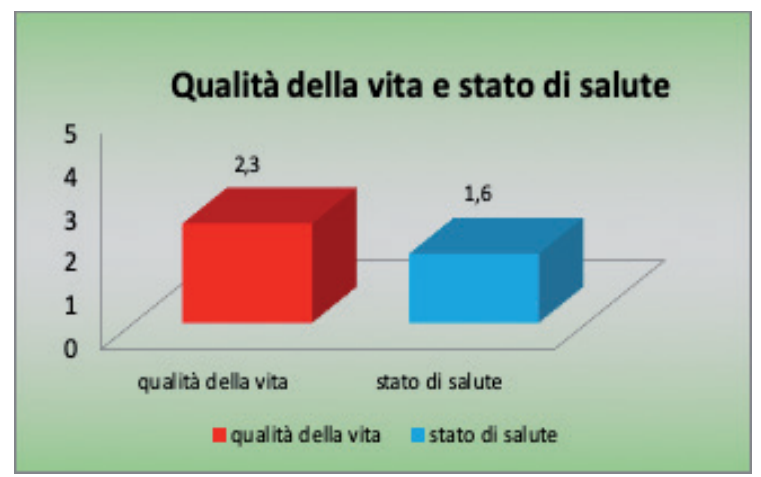

Figura 15. 0: bassa qualità della vita, 5: alta qualità della vita 
mento che coinvolge la mucosa orale, derivante da iperproduzione di collagene. L'esame capillaroscopico è risultato un metodo molto stimolante evidenziare come le alterazioni microvascolari influiscano sulla malattia parodontale nei pazienti affetti da Sclerosi Sistemica.

Il grado di inabilità dell'esercizio delle attività funzionali che coinvolgono gli arti superiori ed inferiori varia a seconda della gravità della patologia, ma in genere sono stati riscontrati valori più alti nelle domande riguardanti gli arti superiori. Tale dato deriva dalla frequente compromissione delle dita delle mani dovuta al fenomeno di Raynaud, spesso associato ad ulcere dolenti, edema, impaccio funzionale e riduzione della flessibilità della cute delle estremità con relative e deducibili ripercussioni sulla possibilità di utilizzare correttamente i presidi per l'igiene orale.

Come dimostra la ricerca effettuata da Mura G. e co 2012 presso l'Università di Cagliari (46), lo stato psicologico dei pazienti risulta alterato. Molto spesso essi perdono le speranze riguardo al futuro, alcuni rifiutano di accettare la patologia ed il grado di ansia e depressione è in genere medio. Chiudendosi in se stessi risulta difficile sia per pazienti che per i familiari migliorare il coping, ovvero "“accettazione della malattia" e "richiesta di aiuto".

A conferma dello studio effettuato da Hyphantis

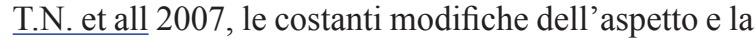
progressiva invalidità hanno un'importante influenza sulla qualità della vita, riducendola considerevolmente (44).

\section{CONCLUSIONI}

Le costanti modificazioni, l'imprevedibile evoluzione della malattia, la progressiva disabilità e le difficoltà fisiche nel mantenere un' igiene orale adeguata rendono estremamente importante il ruolo dell'igienista dentale nell'ambito della prevenzione.

Sulla base dei dati emersi, risulta evidente quanto sia fondamentale la collaborazione dell'igienista dentale con il reumatologo nell'ambito preventivo-terapeutico. Il ruolo dell'igienista in questo caso varia dall'attività clinica che lo vede attuare metodiche preventive primarie e secondarie, all'attività di educazione sanitaria (istruzione sulle tecniche di spazzolamento più idonee, indicazioni sulle norme di un'alimentazione razionale ai fini della tutela della salute dentale) e coinvolgimento nei piani di educazione specifici per questa tipologia di pazienti.

L'introduzione di un programma di profilassi delle patologie dento-parodontali al fine di evitare un peggioramento della qualità della vita che nei pazienti affetti da SSc risulta notevolmente compromessa, si sposa perfettamente con l'obiettivo di migliorare anche la salute sistemica, nonché l'estetica e l'autostima.

Diffondere la cultura preventiva in relazione alla Sclerosi Sistemica, sconosciuta ancora a tanti ma in realtà sempre più diffusa, sia nella popolazione che tra gli operatori, è uno dei principali obiettivi per il futuro. L'interdisciplinarietà e la sinergica collaborazione tra le diverse figure professionali rappresenta un elemento di sicuro successo per garantire un'adeguata qualità di vita anche in presenza di gravi patologie.

\section{REFERENCES}

1. Seibold J. Scleroderma. In: Harris E, Budd RC, Firestein GS, et al. editors. Kelly's testbook of Rheumatology. 7th ed. Philadephia: Elsevier; 2005; 1279-1308

2. Wigley FM, Hummer LK. Clinical features of systemic sclerosis. In: Hocherg M, Silman AJ, Smolen JS, Weinblatt MF, Weisman MH eds. Rheumatology, 3rd ed Philadelphia Elsevier, 2003;146-80.

3. Cozzi F. Sclerosi Sistemica (Sclerodermia). In: Todesco S, Gambari PF. Malattie Reumatiche. 4 Milano Mc Graw Hill; 2007.242-252.

4. Valentini G, Medsger TA jr, Silman AT, Bombardieri S. Eds. Assesment of the patient with systemic sclerosis. Clin Exp Rheumatol 2003; 21(suppl. 29): S1-56

5. Betterle C., Cozzi F. Sclerodermia. In: Le malattie autoimmuni Padova: Piccin Nuova Libraria, 2001; $487,494$.

6. Valentini G. Classification of systemic sclerosis. Clinics in Dermatology 1994; 12: 217-223

7. LeRoy EC, Black C, Fleischmajer R, Jablonska S, Krieg T, Medsger TA jr, et al. Scleroderma (systemic sclerosis): classification, subset and pathogenesis. J Rheumatol 1998 Feb; 15(2): 202-205.

8. Clements PJ, Furst DE eds. Systemic Slcorosis. 2nd Edition Lippincott Williams and Wilkins. Philadelphia 2004: $1-412$.

9. Clements P, Lachenbruch P, Seibold J, Wigley FM. Inter and intra observer variability of total skin thickness score (modified Rodnan) in systemic sclerosis (SSc). J Rheumatol 1995; 22:1281-5.

10. Valentini G, Migliaresi S, La Montagna G. Sclerosi Sistemica. In: Sclerosi Sistemica Napoli: UNIREUMA, IdelsonGnocchi 2008, 177-191.

11. Wigley F. Systemic sclerosis: clinical features. In "Rheumatology” Eds: Klippel JH and Dieppe PA. Masby 1998; 7.9.1.

12. Bryan C, Howard Y, Brennan P, Black C, Silman A. Survival following the onset of scleroderma: results from a restrospective inception cohort study of the UK patient population. Br J Rheumatol 1996 Nov; 35(11): 1122-26.

13. Medsger TA jr, Masi AT, Rodnan GP, Benedek TG, Robinson H. Survival with systemic sclerosis (scleroderma). A lifetable analysis of clinical and demographic factor in 309 patients. Ann Intern Med 1971 Sep; 75(3): 369-76.

14. Tolle SL. Scleroderma: considerations for dental hygienists. Int J Dent Hyg. 2008 May;6(2):77-83.

15. Humprey SP, Russel T. Williamson. A review of saliva: normal composition, flow and function. J Prosthet Dent 2001; $85: 162-9$ 
16. Eliasson L, Carlén A, Almståhl A, Wikström M, Lingström P. Dental plaque $\mathrm{pH}$ and microorganisms during hyposalivation. J Dent Res 2006;85(4):334-8.

17. Parvinen T, Larmas M. The relation of stimulated salivary flow rate and $\mathrm{pH}$ to Lactobacillus and yeast concentrations in saliva. J Dent Res 1981;60(12):1929-35.

18. Guobis Ž, Kareivienė V, Basevičienė N, Paipalienė P, Niedzelskienė I, Sabalys G, Kubilius R, Gervickas A Medicina (Kaunas). Microflora of the oral cavity in patients with xerostomia. 2011;47(12):646-51.

19. Hebbar M, Janin A, Huglo D, Copin MC, Lafyatis R, Devulder B, et al. Xerostomia in systemic sclerosis: systematic evaluation in scintigraphy and lip biopsy in thirty-four patients. Arthritis Rheum 1994; 37(3): 439-41

20. Fisher DJ, Patton LL. Scleroderma: oral manifestations and treatement challenges. Spec Care Dentist 2000; 20(6): 2404.

21. Albilia JB, Lam DK, Blanas N, Clokie CM, Sándor GK.: Small mouths ... Big problems? A review of scleroderma and its oral health implications. J Can Dent Assoc. 2007 Nov;73(9):831-6.

22. Karoui S, Serghini M, Ghouma M, Zouiten L, Matri S, Ben Ghorbel I, Kallel L, Boubaker J, Houmane H, Filali A. [Esophageal motility abnormalities in systemic sclerosis:frequency and predictive factors]. Tunis Med. 2009 Aug;87(8):511-5.

23. Valletta G. La carie dentaria. In: Valletta G. Clinica Odontostomatologica 3 Ed Medica Salernitana. 1994; 128-56

24. Poole JL, Brewer C, Rossie K, Good CC, Conte C, Steen V. Factors related to oral hygene in persons with scleroderma. Int J Dent Hyg. 2005 Feb;3(1):13-7.

25. Chu CH, Yeung CM, Lai IA, Leung WK, Mok MY. Clin Oral Investig. Oral health of Chinese people with systemic sclerosis. 2011 Dec;15(6):931-9.

26. M.R. Benfenati, V.C.Ardizzone. Parodontite. In: Abbinante A., Bresciano S., Camorali C. Igiene Orale Professionale Milano Masson.2001; 13-32

27. M.Calandriello, G.Carnevale, G.Ricci Patogenesi della malattia parodontale in: Parodontologia Bologna Ed. Martina 2006; 113-29

28. Prime SS, Pring M, Davies M, Paterson IC. TGF-beta signal transduction in oro-facial health and non-malignant disease (part I). Crit Rev Oral Biol Med. 2004 Nov 1;15(6):324-36.

29. Ahathya RS, Deepalakshmi D, Emmadi P. Systemic sclerosis. Indian J Dent Res. 2007 Jan-Mar;18(1):27-30.

30. Corrêa MC, Lerco MM, Henry MA. Study in oral cavity alterations in patients with gastroesophageal reflux disease. Arq Gastroenterol. 2008 Apr-Jun;45(2):132-6.

31. Serra MC, Messias DC, Turssi CP. Braz Oral Res. Control of erosive tooth wear: possibilities and rationale. 2009; 23 Suppl 1:49-55.

32. Tantbirojn D, Pintado MR, Versluis A, Dunn C, Delong R.Quantitative analysis of tooth surface loss associated with gastroesophageal reflux disease: a longitudinal clinical study. J Am Dent Assoc. 2012 Mar;143(3):278-85.

33. Doucet JC, Morrison AD. Bilateral mandibular condylysis from systemic sclerosis: case report of surgical correction with bilateral total temporomandibular joint replacement. Craniomaxillofac Trauma Reconstr. 2011 Mar; 4 (1):11-8.

34. F.Gombos, R. Serpico Sclerosi Sistemica progressiva In: Immunopatologia Orale Piccin 1999; 293-69

35. Scardina G.A., Carini F., Leone A., Valenza V, Fuca G., Messina P. Panoramic Periodontal Capillaroscopy- a New Diagnostic Method. Dent. Med. Probl. 2004, 41, 3, 449-453.

36. Scardina G.A., Carini F., Messina P. Oral capillaroscopy: a new diagnostic method Reumatismo, 2005; 57(4):295-304

37. Scardina G.A., Ruggieri A., Messina P; Oral microcirculation observed in vivo by videocapillaroscopy: a review. Journal of Oral Science Vol. 51, 2009; 1-10.

38. Scardina GA, Pizzigatti ME, Messina P. Periodontal microcirculatory abnormalities in patients with systemic sclerosis. J Periodontol. 2005 Nov; 76(11):1991-5.

39. Nguyen C, Bérezné A, Baubet T, Mestre-Stanislas C, Rannou F, Papelard A, Morell-Dubois S, Revel M, Guillevin L, Poiraudeau S, Mouthon L; Groupe Français de Recherche sur la Sclérodermie. Association of gender with clinical expression, quality of life, disability, and depression and anxiety in patients with systemic sclerosis. PLoS One. 2011 Mar 9;6(3):e17551.

40. Thombs BD, Hudson M, Bassel M, Taillefer SS, Baron M; Canadian Scleroderma Research Group. Sociodemographic, disease, and symptom correlates of fatigue in systemic sclerosis: evidence from a sample of 659 Canadian Scleroderma Research Group Registry patients. Arthritis Rheum. 2009 Jul 15;61(7):966-73.

41. Thombs BD, Taillefer SS, Hudson M, Baron M.Depression in patients with systemic sclerosis: a systematic review of the evidence. Arthritis Rheum. 2007 Aug 15;57(6):1089-97.

42. Thombs BD, Hudson M, Taillefer SS, Baron M; Canadian Scleroderma Research Group.Prevalence and clinical correlates of symptoms of depression in patients with systemic sclerosis. Arthritis Rheum. 2008 Apr 15;59(4):504-9.

43. Nguyen C, Poiraudeau S, Mestre-Stanislas C, Rannou F, Bérezné A, Papelard A, Choudat D, Revel M, Guillevin L, Mouthon L.Employment status and socio-economic burden in systemic sclerosis: a cross-sectional survey. Rheumatology (Oxford). 2010 May;49(5):982-9.

44. Hyphantis TN, Tsifetaki N, Siafaka V, Voulgari PV, Pappa C, Bai M, Palieraki K, Venetsanopoulou A, Mavreas $\mathrm{V}$, Drosos AA. The impact of phychological functioning upon systemic sclerosis patient's quality of life. Semin Arthritis Rheum. 2007. Oct;(2):81-92.

45. Beretta L. Astori S., Ferrario E., Caronni M., Raimondi M., Scorza R. Determinants of depression in 111 italian patients with systemic sclerosis. Reumatismo, 2006; 58(3):219-225.

46. Mura G, Bhat KM, Pisano A, Licci G, Carta M.Psychiatric symptoms and quality of life in systemic sclerosis. Clin Pract Epidemiol Ment Health. 2012;8:30-5.

47. Miranda S1, Armengol G1, Le Besnerais M1, Lévesque H1, Benhamou Y2.New insights into systemic sclerosis related microcirculatory dysfunction by assessment of sublingual micr locirculation and vascular glycocalyx layer. Results from a preliminary study. Microvasc Res. 2015 